\author{
نسيبه عبدالهزاده'، رضا فتوتَّ، فريد شكارى 'و سيد محمد علوى سينىع
}

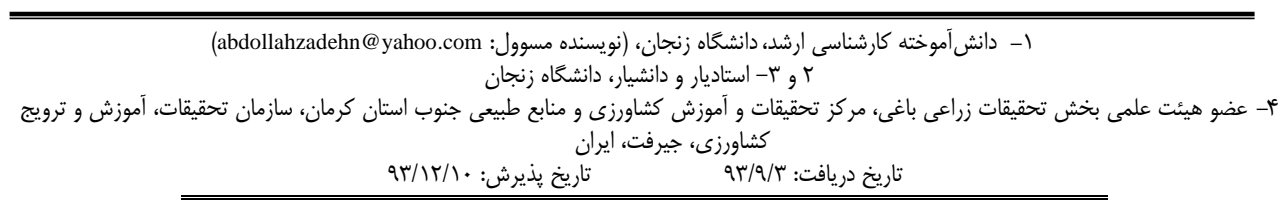

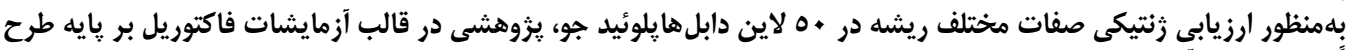

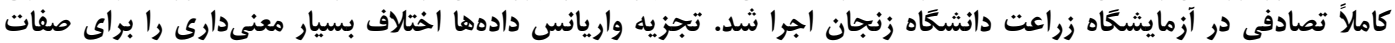

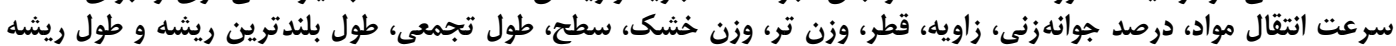

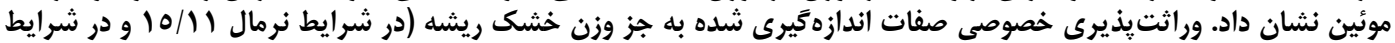

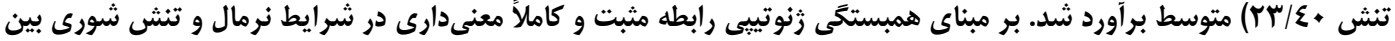

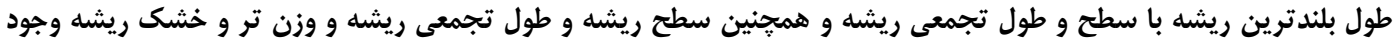

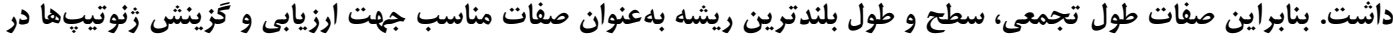
برنامههاى به نزادى توصيه مى مَردند.

وازههاى كليدى: جو، شورى، وراثتيذيرى خصوصى، همبستكى زنوتيبى

إلىثنها و يك زن اصلى كه طول دورهى رشد رويشى رادئ

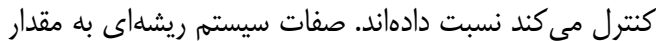

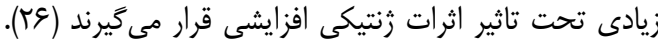

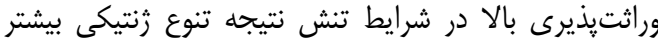

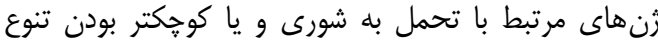

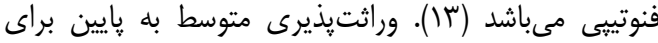

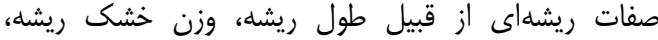

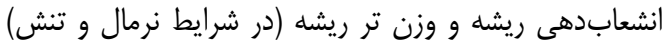

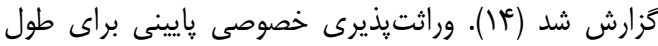

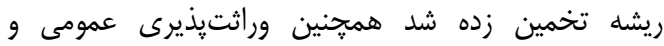

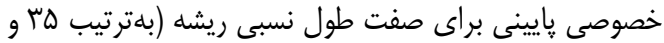

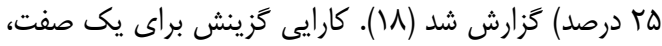

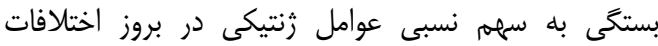

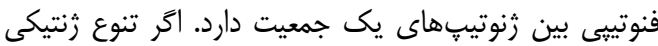

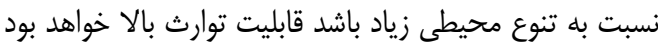

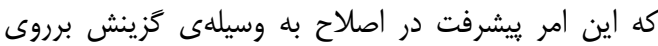

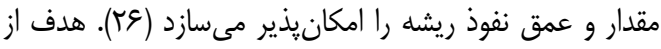

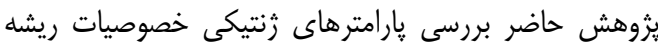

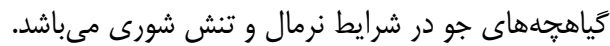

\section{مواد و روشها}

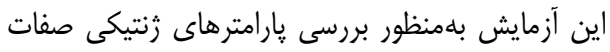

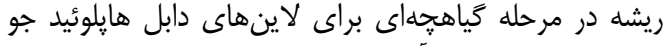

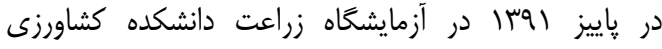

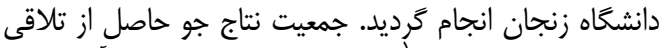

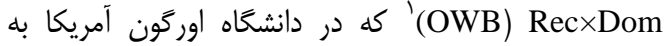

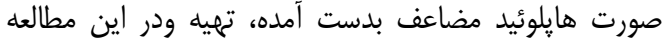
مورد بررسى قرار كرفت. ينجاه لاين دابل هائلئئيد از بين اين آنائ
رشد و نمو گياهان تحت تأثير خصوصيات زنتيكى و

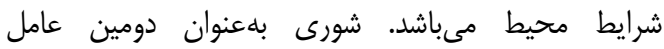

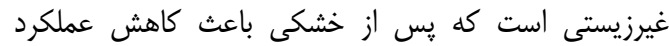

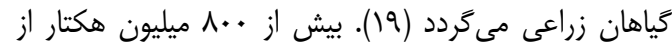

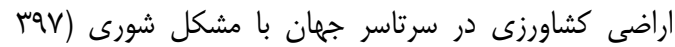

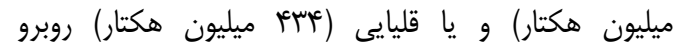

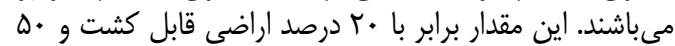

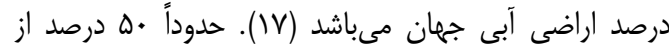
سطح زير كشت محصولات كشاورزى در ايران به به دلي دليل قرار

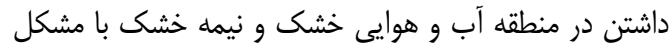

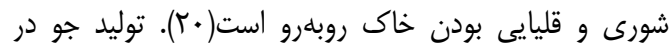

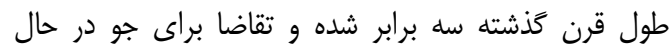

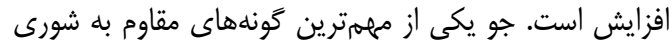

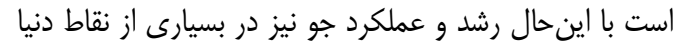

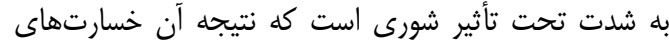

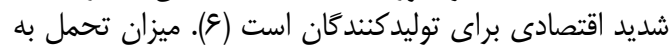

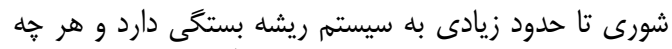

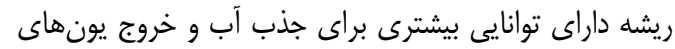

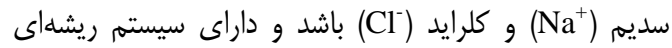

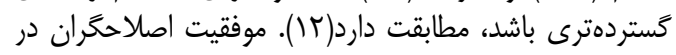

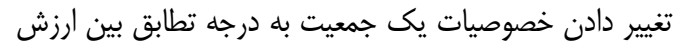

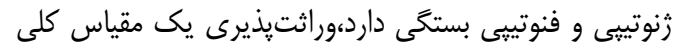

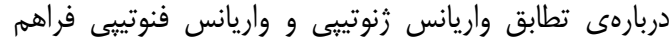

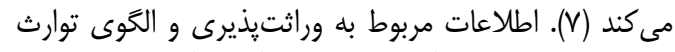

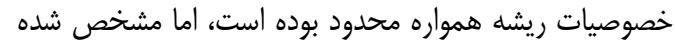
است كه اين صفات بوسيله يك سيسته هنداره

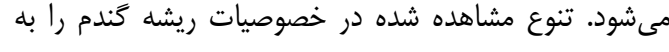


ضريب تغييرات زنتيكى و فنوتييى نيز با استفاده از فرمولهاى زير محاسبه گرديد.

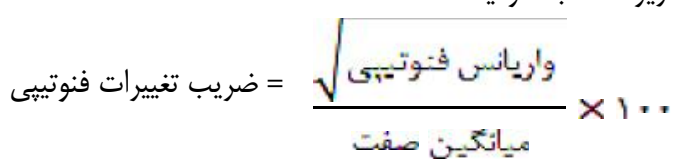

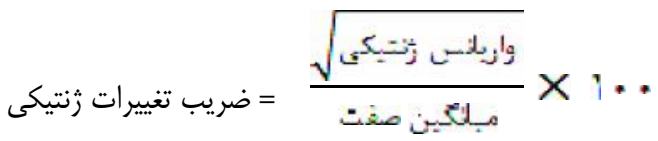

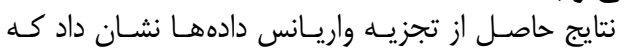

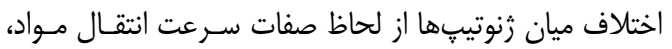

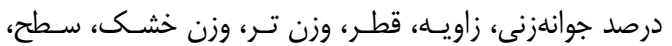

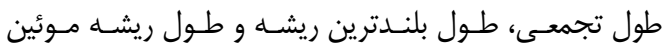

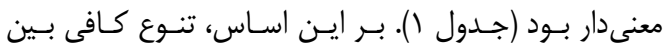

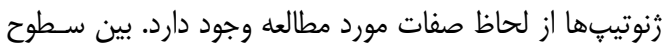

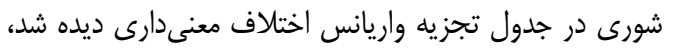

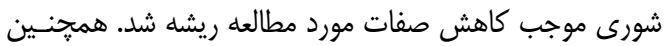

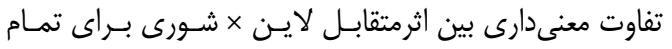

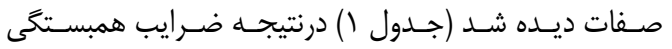

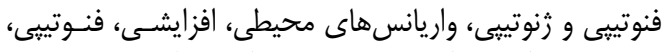

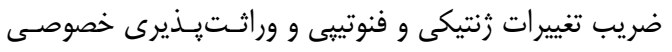

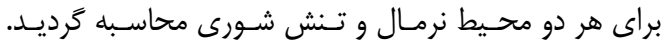

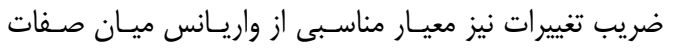

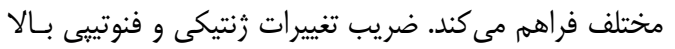

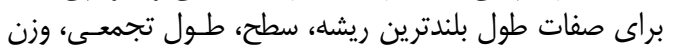

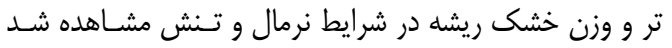

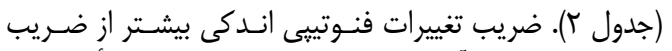

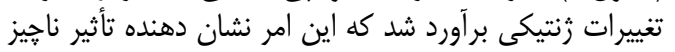

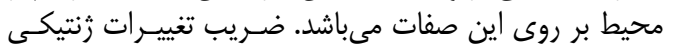

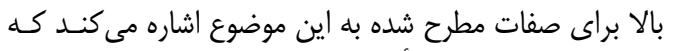

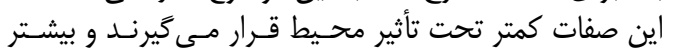

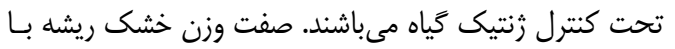

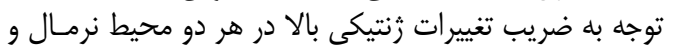

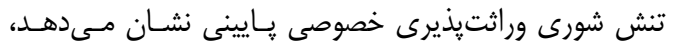

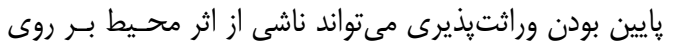

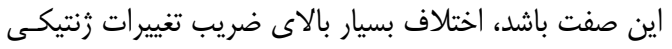

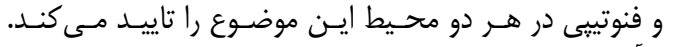

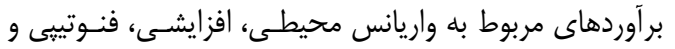

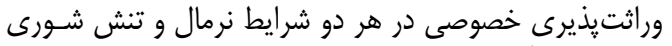

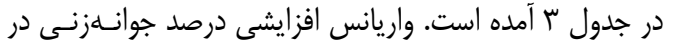

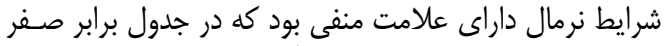

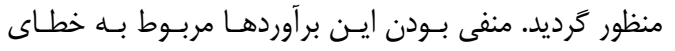

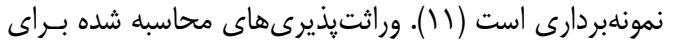

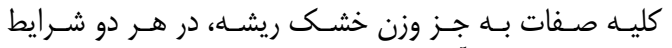

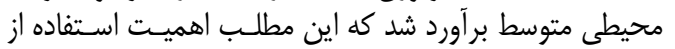

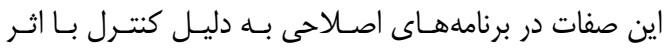

جمعيت مورد استفاده قرار گرفتند. اين لاينها تحت شرايط

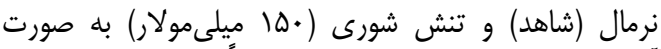

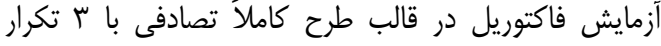

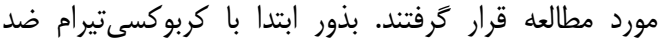

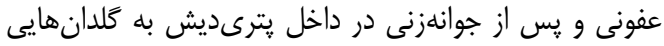

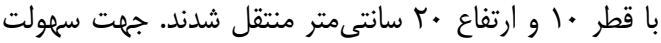

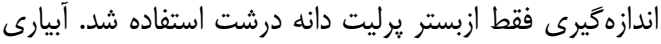

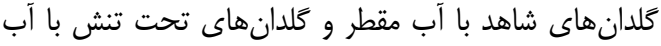

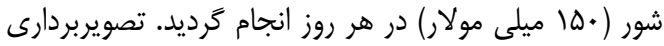

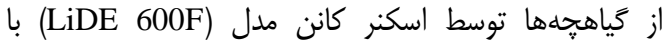

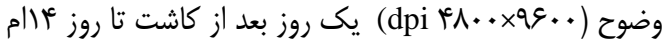

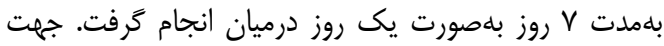

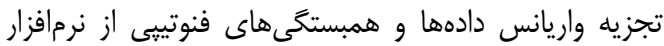
SPSS

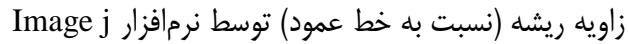

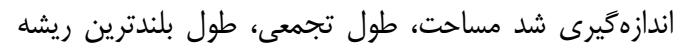

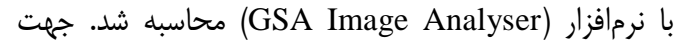
محاسبه سرعت انتقال مواد، بذور درون ظروف فروف يترى كشت

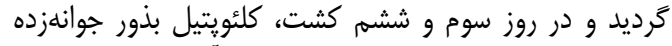

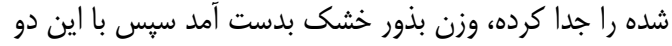

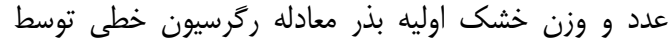

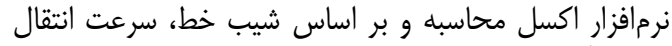

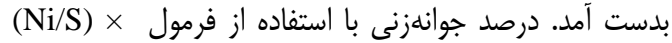
محاسبه گرديد. در اين فرمول GP = 100

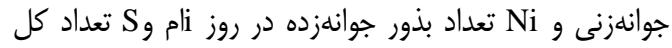

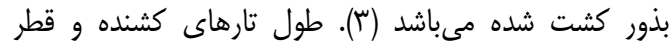

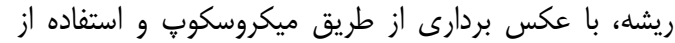

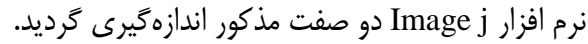

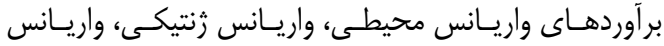

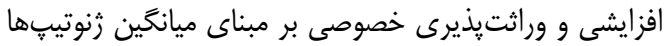

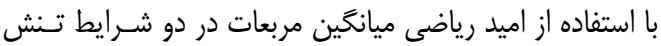

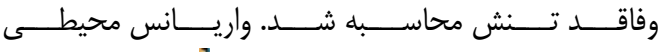

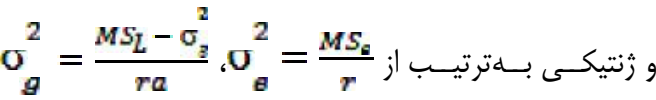

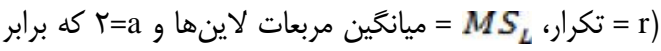
سطح تنش و شاهد است) و واريـانس افزايشـى بــا اسـتفاده از فرمول واريـانس زنتيكـى وارثـتِـــيرى خصوصـى محاسـبه گرديـــ ( $\left.h_{N}^{2}=\frac{{ }_{0}^{2}}{\frac{\pi}{2}}\right)$

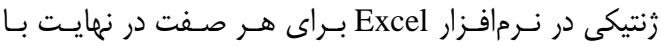

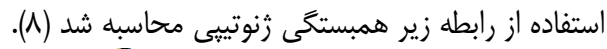

$$
\mathrm{r}_{g}=\frac{\sigma_{\mathrm{Exy}}^{2}}{\sqrt{\sigma_{\mathrm{gx}}^{2} \times \mathrm{\sigma}_{\mathrm{gy}}^{2}}}
$$


ترتيب و9/ • تا 9/ • تخمين زده شد در صورتى كـه در شـرايط

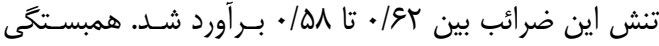

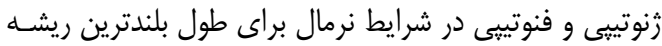

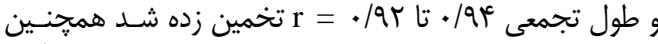

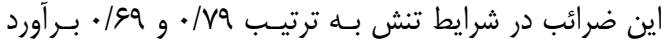

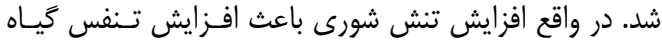

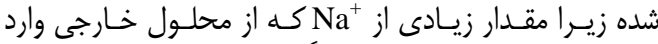

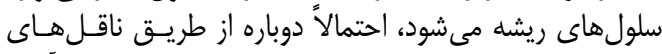

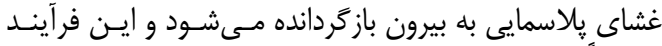

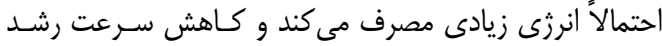

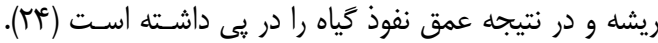

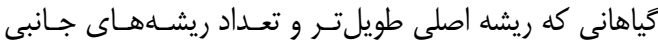

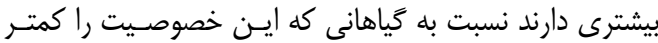

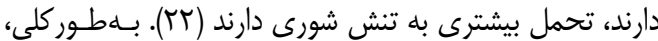

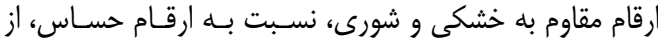

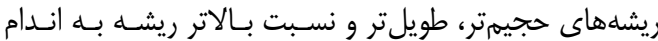

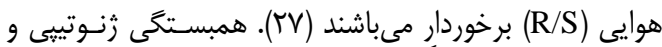

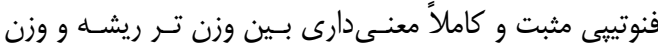

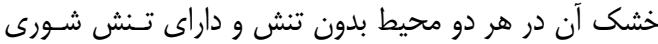

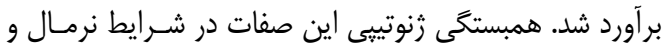

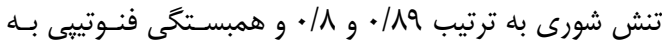

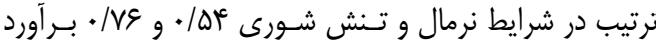

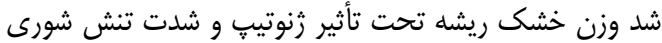

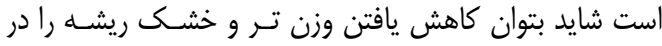

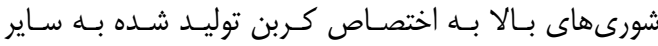

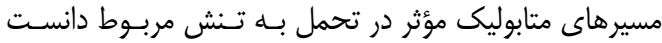

در اين آزمايش همبستخى زنوتييى مثبت و معنى مارى

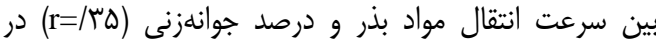

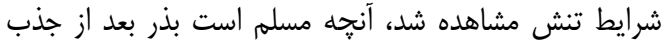

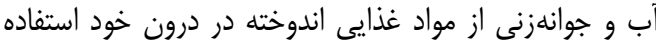

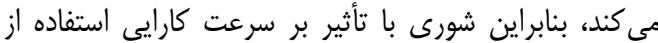

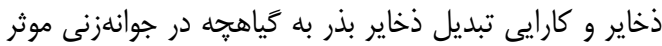

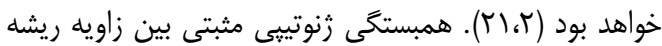

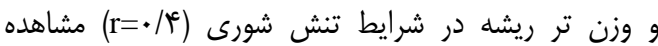

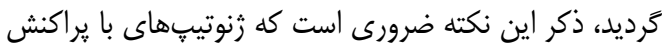

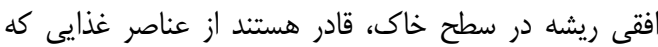

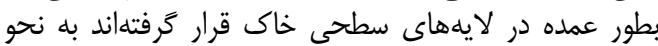

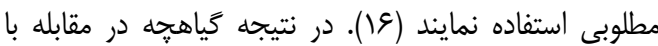

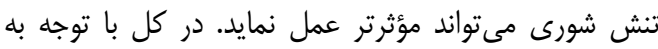

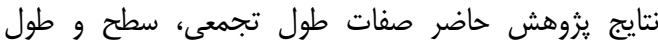

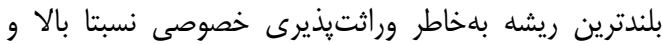

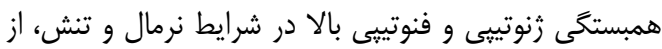

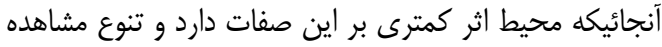

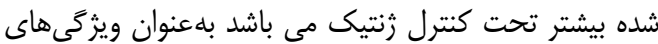

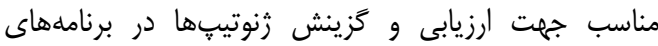

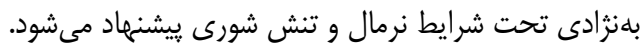

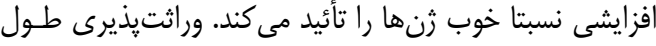

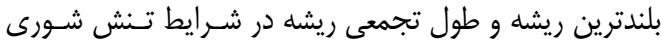

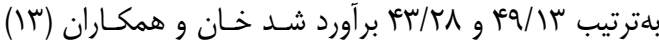

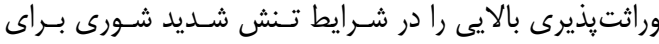

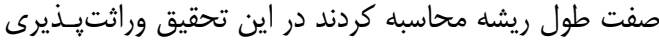

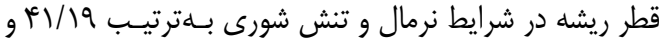

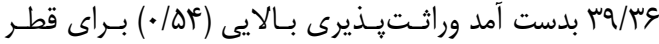

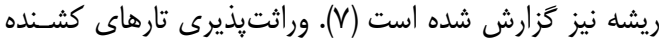

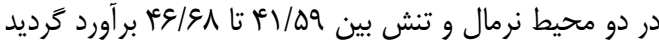

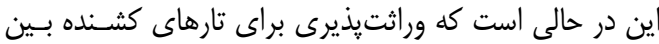

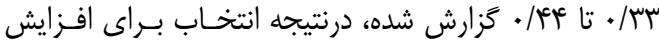

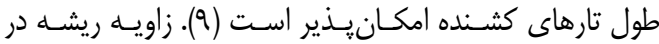

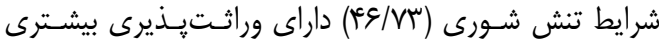

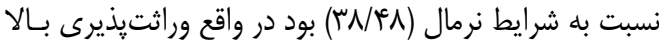

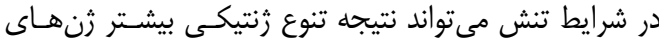

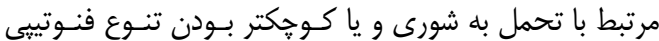

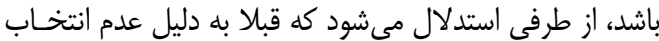

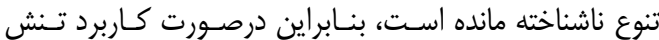

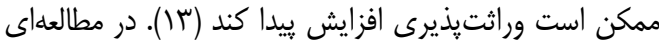

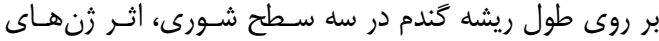

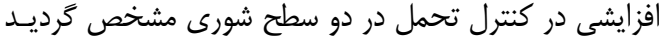

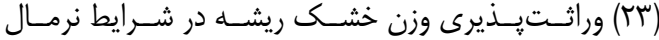

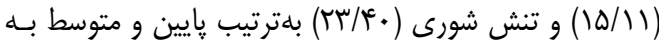

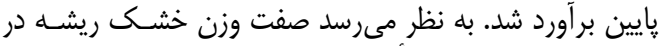

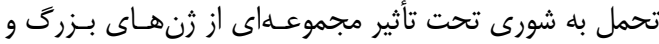

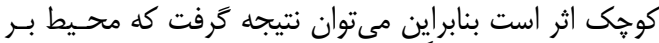

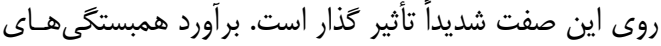

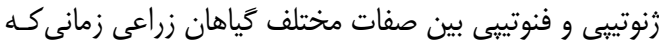

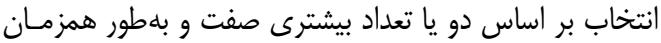

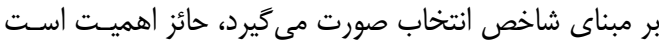

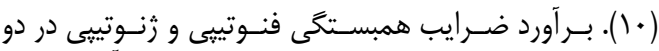

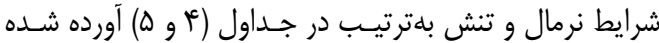

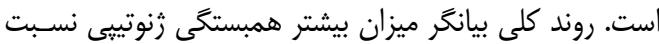

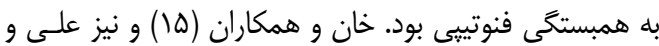

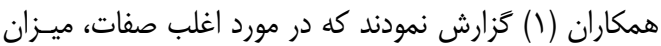

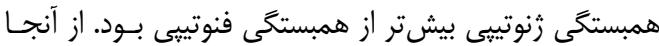

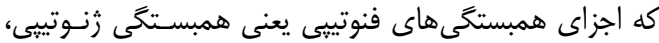

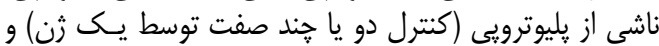

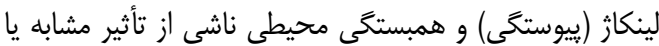

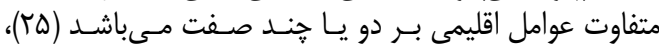

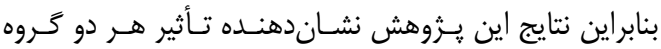

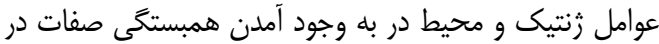

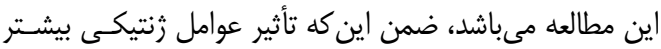

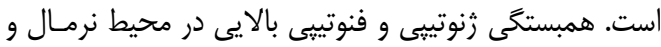

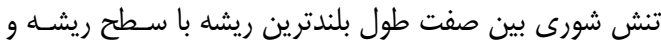

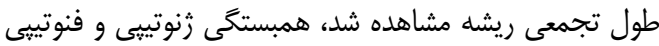

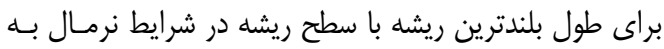


Table 1. Analysis of variance root traits in barley lines under salt stress

جدول ا- نتايج تجزيه واريانس صفات مورد بررسى ريشه در لاينهاى جو تحت تنش شورى

\begin{tabular}{|c|c|c|c|c|c|c|c|c|c|c|c|}
\hline \multicolumn{10}{|c|}{ ميانكين مربعات } & \multirow[b]{2}{*}{ آزادى درجه } & \multirow[b]{2}{*}{ منابع تغييرات } \\
\hline وزن خشك & وزن تر & طول تجمعى & سطح & طول ريشه & طول بلندترين ريشه & قطر ريشه & جوانهزنى & انتقال مواد & زاويه & & \\
\hline $\mid Y N / \Delta T^{\text {䓪 }}$ & $9 q \cdot r / 1 .{ }^{*}$ & $F V / q)^{* * *}$ & $|\varphi \psi / 9|^{* * * *}$ & TVTN/DS & $11 Q \cdot V / / \Delta r^{* *: *}$ & 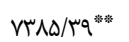 & 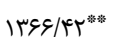 & 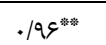 & gFr/l": & 19 & لاين \\
\hline 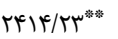 & $r \cdot T \cdot V V / \Lambda e^{* * *}$ & $\pi \cdot \Delta / \omega^{* * *}$ & 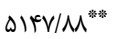 & $119 \% / 19^{* * *}$ & GTYYADI/N" & $11199 / .9^{* * *}$ & $\mid \Lambda T \Delta / r^{\prime *}$ & $1 / \mu \cdot *$ & 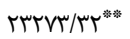 & 1 & شورى \\
\hline$\Lambda \Delta / \mu \Lambda^{*}$ & f) $99 / V^{*}$ & 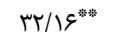 & 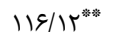 & 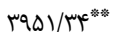 & NEYVY/qV **: & $\Delta ৭ ৎ \varnothing / \Delta)^{*}$ & 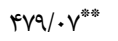 & $\cdot \mid 9 \Lambda^{* *: 3}$ & 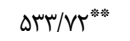 & pq & لاين × شورى \\
\hline $9 \cdot 119$ & $1 \cdot V r / 9 q$ & $r \cdot / \Lambda F$ & $r / \cdot 1$ & $r I I / V$ & rAr/av & 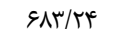 & $\Delta \Delta$ &.$/ 11$ & DI/R & $r .$. & اشتباه آزمايشى \\
\hline$N / 91$ & ل & $\Delta / \cdot V$ & $1 / \Delta$ & $\mid 9 / 19$ & IN/VT & $r q / \cdot r$ & אוא & ع ع/. & V/QD & - & ضريب تغييرات (\%) \\
\hline
\end{tabular}

جدول r- ضريب تغييرات زنتيكى و فنوتيبى در شرايط فاقد تنش (اعداد بالايى) و شرايط تنش (اعداد بإينى) Table 2. The coefficient of genetic and phenotypic variations in non-stress conditions (top numbers) and stress condition (low numbers)

\begin{tabular}{|c|c|c|c|c|c|c|c|c|c|c|}
\hline وزن خشك & وزن تر ريشه & طول تجمعى & ريشح & طول ريشه & طول بلندترين & قطر ريشه & جوانهزنى & سرعت انتقال & زاويه & صفات \\
\hline$\Delta V / \& \varepsilon$ & $q \% / \cdot \Delta$ & $9 I / T r$ & $\Delta S / \mu^{\prime} \Lambda$ & TN/DS & $19 / 99$ & $T Y / \cdot \Delta$ & $T r / Q D$ & $\mu \cdot / \Lambda \mu$ & $\mid F / T r$ & \multirow[b]{2}{*}{ ضريب تغييرات زنتيكى } \\
\hline$r q / T V$ & 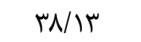 & $\Delta \cdot / 4 \mathrm{~V}$ & $\Delta \cdot / \mu \Lambda$ & $r \cdot / / \Delta$ & & $1 \% / q F^{\circ}$ & $r \mu / \Delta V$ & TI/DT & 19/94 & \\
\hline$V F / \Delta T$ & $8 N / 11$ & $g r / 4$. & $\left.\Delta V / F^{c}\right)$ & rT/G. & FV/IT & $\Gamma \varepsilon / \Delta V$ & TF/M & "N/ & $|Q / T|$ & \multirow[b]{2}{*}{ ضريب تغيييرات فنوتييى } \\
\hline$g / / 9 \mid$ & $\Delta F / \Delta D$ & $\Delta \Delta / \wedge \mu$ & $\Delta \mu / \cdot q$ & II/VI & $r / v$ & $|V / V|$ & rळ/^q & $r N / v$. & $18 / \mathrm{V}^{\mathrm{N}}$ & \\
\hline
\end{tabular}


جدول س- برآورد واريانس محيطى، واريانس افزايشى، واريانس فنوتيبى و وراثتيذيرى خصوصى (بر مبناى ميانگين زنوتيبها) صفات ريشه

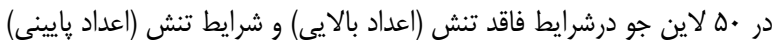

Table 3. The estimated environmental variance, additive variance, phenotypic variance and Narrow sense heritability (based on the average genotypes) root traits in barley lines 50 non-stress conditions (top numbers) and stress conditions (low numbers)

\begin{tabular}{|c|c|c|c|c|}
\hline$h_{N}^{2}$ & $\sigma_{\mathrm{ph}}^{2}$ & $\sigma_{\mathrm{A}}^{2}$ & $\sigma_{e}^{2}$ & صفت \\
\hline$r N / F \wedge$ & ITK//R & FV/s. & NT/qr & \multirow{2}{*}{ زاويه ريشه } \\
\hline & $\Lambda q / \mu \mathrm{re}$ & $F / N \Delta$ & $\mid V / \Delta F$ & \\
\hline$p q / \Lambda 1$ & TMQYN/TD & $\mid N F / \Delta / \cdot \Delta$ & TTK/FY & \multirow{2}{*}{ طول بلندترين ريشه } \\
\hline$r q / 1 \Gamma$ & $F v / f / e q$ & THIS/FD & TYY/DT & \\
\hline$\Gamma N / N$ & $11 / \Delta V$ & D/G & $\cdot / \mathrm{M}$ & \multirow{2}{*}{ طول تجمعى ريشه } \\
\hline$r \mu / r \Lambda$ & $r / . \Delta$ &.$/ 19$ & $\cdot / \Lambda$. & \\
\hline$\lceil N / \lambda$. & $r V / \Delta$ & $\mid N / \mu$. & $r / 99$ & \multirow{2}{*}{ سطح ريشه } \\
\hline$\Leftrightarrow g / \Delta V$ & $8 / 48$ & $r / .1$ & $1 / \pi r$ & \\
\hline$F 1 / \Delta q$ & SID/DF & $T \Delta S / .9$ & $r 1 . / T^{c}$ & \multirow{2}{*}{ طول ريشه موئين } \\
\hline $\mid \& / 91$ & $\Delta S N / T I$ & $r Y_{D} /{ }^{\prime}$. & $11 \% / 19$ & \\
\hline $41 / 19$ & $|1| 1 / 9$. & $V^{*} \in / N V$ & $9 \Delta V / \Delta)$ & \multirow[b]{2}{*}{ قطر ريشه } \\
\hline$r q / r q$ & $94 / / 4$ & $r \Delta T / F i$ & $r+N / q V$ & \\
\hline$\mu \kappa / q)$ & $1 / \Delta \cdot / 99$ & 94.199 & $\mid V \cdot V / \Delta f^{f}$ & \multirow{2}{*}{ وزن تر ريشه } \\
\hline$r q / \mu \varphi$ & $r \Delta F / T r$ & $1.4 / q r$ & $r+\Delta / \Delta \Delta$ & \\
\hline $10 / 11$ & $\Delta r / N G$ & $\mathrm{~N} \cdot 9$ & $111 / V 1$ & \multirow{2}{*}{ وزن خشك ريشه } \\
\hline$r \pi / \kappa$. & D/99 & $1 / 49$ & N/GT & \\
\hline$\cdot$ & .1 .9 & . & $\cdot \pi r$ & \multirow[b]{2}{*}{ درصد جوانه زنى } \\
\hline$\kappa \psi / \Delta F$ &.$\mu r$ & .1 .1 & .1 .1 & \\
\hline$\mu q / \Delta V$ &.$/ 14$ & $\% \cdot \Delta$ &.$/ T T$ & \multirow{2}{*}{ سرعت انتقال مواد } \\
\hline $\mathrm{rV} / \mathrm{Qu}$ & זו/. & $.1 . \Delta$ & .111 & \\
\hline
\end{tabular}

جدول عا- ضرايب همبستخى زنوتييى (اعداد بالاى قطر) و همبستخى فنوتييى (اعداد پائين قطر) در شرايط نرمال

Table 4. Genotype correlation coefficients (numbers above the diameter) and phenotypic correlation (lower numbers in diameter) under normal conditions

\begin{tabular}{cccccccccc}
\hline \\
\hline
\end{tabular}

جدول ه- ضرايب همبستخى زنوتييى (اعداد بالاى قطر) و همبستخى فنوتييى (اعداد پائين قطر) در شرايط تنش

Table 5. Genotype correlation coefficients (numbers above the diameter) and phenotypic correlation (lower numbers in diameter) under stress conditions

\begin{tabular}{|c|c|c|c|c|c|c|c|c|c|c|}
\hline وزيش خشك & وزن تر ريشه & طول تجمعى & سطح ريشه & طولئ ريشه & بلندترين & قطر ريشه & جواندزنى & سرعت انتقال & زاويه ريشه & \\
\hline$\cdot / T \Lambda^{*}$ & $\cdot / c^{* m}$ & $\cdot / T^{\text {ns }}$ & $\cdot / r^{\mathrm{nS}}$ & $-\cdot / / \lambda^{\mathrm{nS}}$ & $\cdot 1 \cdot 0^{\text {ns }}$ & $\cdot / / \omega^{\mathrm{nS}}$ & $\cdot / \cdot r^{\text {ns }}$ & $\cdot / 1 \varphi^{\mathrm{ns}}$ & 1 & زاويه ريشه \\
\hline$\cdot / r^{\mathrm{ns}}$ & $\cdot(r)^{\mathrm{ns}}$ &.$/ .9^{\mathrm{ns}}$ & $\cdot / 11^{\mathrm{ns}}$ & $-\cdot / 9^{\text {ns }}$ & $\cdot / 1^{\mathrm{ns}}$ & $\cdot / / v^{\mathrm{ns}}$ &.$/ \% \omega^{*}$ & 1 & $\cdot / / r^{\mathrm{ns}}$ & سرعت انتقال \\
\hline $\begin{array}{l}\cdot / 1^{\mathrm{ns}} \\
\cdot / \cdot 8^{\mathrm{ns}}\end{array}$ & $\begin{array}{l}-\cdot / \cdot 1^{\mathrm{ns}} \\
-\cdot / \cdot 0^{\mathrm{ns}}\end{array}$ & $\begin{array}{l}. / 1 r^{\mathrm{ns}} \\
. / \mu r^{4}\end{array}$ & $\begin{array}{l}\cdot / \mathrm{f}^{\mathrm{ns}} \\
\cdot / \mathrm{rq}^{*}\end{array}$ & $\begin{array}{l}-\cdot / \cdot 1^{\mathrm{ns}} \\
\cdot / /^{\mathrm{ns}}\end{array}$ & $\begin{array}{l}\cdot / \cdot \wedge^{\mathrm{ns}} \\
\cdot / \wedge \Delta^{\mathrm{ns}}\end{array}$ & $\begin{array}{c}\cdot \pi \omega^{*} \\
1\end{array}$ & ${ }^{1}$ & $\begin{array}{l}. / r \Delta^{\circ} \\
. / / r^{\mathrm{ns}}\end{array}$ & $\begin{array}{l}\cdot / \cdot \mathrm{rns}^{\mathrm{ns}} \\
\cdot / \mathrm{N}^{\mathrm{ns}}\end{array}$ & قرصر ريشه جوانهزنى \\
\hline$-\cdot / r^{\mathrm{ns}}$ & $-.1 \cdot 9^{\mathrm{ns}}$ & $\cdot / Q^{* * s}$ &. $\mid q \varphi^{* s s}$ & $\cdot / r^{\mathrm{ns}}$ & 1 & $\cdot / 1^{\mathrm{ns}}$ & $\cdot / \cdot \mathrm{v}^{\mathrm{ns}}$ & $\cdot 1 \cdot 9^{\mathrm{ns}}$ & $\cdot / \cdot 0^{\mathrm{ns}}$ & طول بلندترين \\
\hline $\begin{array}{l}\cdot / \cdot 0^{\mathrm{ns}} \\
-\cdot / \cdot 0^{\mathrm{ns}}\end{array}$ & $\begin{array}{l}-\cdot / 11^{\mathrm{ns}} \\
\cdot / \cdot 0^{\mathrm{ns}}\end{array}$ & $\begin{array}{l}\cdot / \cdot)^{\mathrm{ns}} \\
. / \mathrm{N}^{\mathrm{ses}}\end{array}$ & $\begin{array}{c}\cdot \cdot \cdot \cdot r^{\mathrm{ns}} \\
1\end{array}$ & $\begin{array}{c}1 \\
-\cdot / \cdot 1^{\mathrm{ns}}\end{array}$ & $\begin{array}{l}\cdot / \mathrm{r}^{\mathrm{ns}} \\
\cdot / \Delta \mathrm{N}^{* * *}\end{array}$ & $\begin{array}{l}. / q^{\mathrm{ns}} \\
. / r^{\mathrm{ns}}\end{array}$ & $\begin{array}{l}-\cdot|9|^{+\infty} \\
\cdot / 10\end{array}$ & $\begin{array}{l}\cdot / \cdot 0^{\mathrm{ns}} \\
\cdot / 1^{\mathrm{ns}}\end{array}$ & $\begin{array}{l}-\cdot / 19^{\mathrm{ns}} \\
\cdot / 18^{\mathrm{ns}}\end{array}$ & ططحل ريشه موئين \\
\hline$\cdot / r^{\mathrm{ns}}$ &.$/ 1 v^{\mathrm{ns}}$ & 1 & . & $\cdot / \cdot \Delta^{\mathrm{ns}}$ &.$/ 89 *$ & $\cdot / \pi r^{n s}$ & $\cdot / \cdot \mathrm{n}^{\mathrm{ns}}$ & $.1 .8^{\mathrm{ns}}$ &.$/ 1 v^{\mathrm{ns}}$ & طول تجمعى \\
\hline $\begin{array}{c}\cdot / \Lambda^{* 0} \\
1\end{array}$ & $\begin{array}{l}1 \\
. / V \xi^{* * s}\end{array}$ & $\begin{array}{l}\cdot / r \Lambda^{*} \\
-* / \cdot r^{\mathrm{ns}}\end{array}$ & $\begin{array}{l}\cdot / \cdot r^{\mathrm{ns}} \\
-\cdot / \cdot r^{\mathrm{ns}}\end{array}$ & $\begin{array}{l}-\cdot / /^{\mathrm{ns}} \\
\cdot / \cdot \cdot \gamma^{\mathrm{ns}}\end{array}$ & $\begin{array}{l}-\cdot / r r^{\mathrm{ns}} \\
-\cdot / \mathrm{h}^{\mathrm{ns}}\end{array}$ & $\begin{array}{l}-\cdot / \cdot r^{\text {ns }} \\
\cdot / \cdot 9^{\text {ns }}\end{array}$ & $\begin{array}{l}\cdot / 1^{\mathrm{ns}} \\
\cdot / 1^{\mathrm{ns}}\end{array}$ & $\begin{array}{l}. / q^{\mathrm{ns}} \\
1 / \mathrm{r}^{\mathrm{ns}}\end{array}$ & $\begin{array}{l}. / r \varepsilon^{*} \\
.|r|^{\text {ns }}\end{array}$ & وزن خشك ريشه ريشه \\
\hline
\end{tabular}


1. Ali, F. B. Sikdar, A.K. Roy and O.I Joarder. 2005. Correlation and genetic variation of different genotypes of lablab bean, (Lablab perpureus L.), Sweet. Bangladesh Journal of Botany, 34: 125-128.

2. Alizadeh, A. 2012. Relationship water and soil and plant. 1th edn. $3^{\text {th }}$ Inc. Mashhad University Jahad publications. Mashhad, Iran, 472 pp (In Persian).

3. Bajji, M., J.M. Kinet and S. Lutts. 2002. Osmotic and ionic effects of $\mathrm{NaCl}$ on germination, early seedling growth, and ion content of Atriplexhalimus (Chenopodiaceae). Canadian journal of botany, 80: 297-304.

4. Bchini, H., M.B. Naceur, R. Sayar, H. Khemira and L.B. Kaab-Bettaeïb. 2010. Genotypic differences in root and shoot growth of barley (Hordeum vulgare L.) grown under different salinity levels. Hereditas. 147: 114-122.

5. Borzouei. A.. M. Kafi. H. Khazaei and M. Mousavi Shalmani. 2012. Effect of irrigation water salinitv on root traits of two salt-sensitive and salt-tolerant wheat cultivars and its relationship with yield in greenhouse. Journal of Science and Technology of Greenhouse Culture, 2: 95-107 (In Persian).

6. Chen, Z., S. Shabala, N. Mendham, I. Newman, G. Zhang and M. Zhou. 2008. Combining ability of salinity tolerance on the basis of NaCl-induced $\mathrm{K}$ flux from roots of barley. Crop Science, 48: 13821388.

7. Dabholkar, A. 1992. Elements of Biometrica Genetics. $1^{\text {st }}$ edn, Ashok Kumar Mittal Ltd. New Delhi, $493 \mathrm{pp}$.

8. Falconer, D.S. 1983. Introduction to Quantitative Genetics. Longman Pub., London.

9. Gahoonia, T.S. and N.E. Nielsen. 2004. Root traits as tools for creating phosphorus efficient crop varieties. Plant and Soil, 260: 47-57.

10. Houshmand, S., A. Arzani and S.A. Maibody. 2004. Influences of drought and salt stress on grain quality of durum wheat. Proceedings of the $17^{\text {th }}$ Eucarpia General Congress, pp: 383-386. Tulln, Austria.

11. Kearsey, M.J. 1993. Biometrical genetics in breeding. In M.D. Hayward, N.O. Bosemark, I. Romagosaand M. Cerezo (Eds.), Plant Breeding, pp: 163-183. Springer Netherlands.

12. Kamrava. S., N.A. Babaeian Jelodar and N.A. Bagheri.. 2016. Evaluation of Some Soybean Genotypes (Glycine max) under Salt Stress. Journal of Crop Breeding, 8: 57-63.

13. Khan, A.A., S.A. Rao and T. McNeilly. 2003. Assessment of salinity tolerance based upon seedling root growth response functions in maize (Zea mays L.). Euphytica, 131: 81-89.

14. Khan, F.A., S. Ali, A. Shakeel, A. Saeed and G. Abbas. 2006. Correlation analysis of some quantitative characters in Brassica napus L. Journal of Agriculture Research, 44: 7-14.

15. Khan, I.A., S. Habib, H.A. Sadaqat and M.H.N. Tahir. 2004. Comparative evaluation and analysis of seedling traits for drought tolerance in maize. International Journal of Agriculture and Biology (Pakistan) 6: 246-251.

16. Manschadi, A.M., G.L. Hammer and J.T. Christopher. 2008. Genotypic variation in seedling root architectural traits and implications for drought adaptation in wheat (Triticum aestivum L.). Plant and Soil, 303: 115-129.

17. Nakhoda, B., H. Leung, M.S. Mendioro, G. Mohammadi-nejad and A.M. Ismail. 2012. isolation, characterization, and field evaluation of rice (Oryza sativa L.) mutants with altered responses to salt stress. Field Crops Research, 127: 191-202.

18. Ortiz, R. and A. Golmirzaie. 2003. Genetic parameters for agronomic characteristics. early and intermediate breeding populations of true potato seed. Hereditas, 139: 212-216.

19. Rengasamy, P. 2010. Soil processes affecting crop production in salt-affected soils. Functional Plant Biology, 37: 613-620.

20. Shahin Kaleybar, B., Gh. Ne'matzadeh., S.H.R. hashemy., H. askary and S. Kabirnotaj. 2013. Physiological and Genetic Responses of Halophyte Aeluropus Littoralis to Salinity. Journal of Crop Breeding, 5(12): 15-29

21. Soltani, A., M. Gholipoor and E. Zeinali. 2006. Seed reserve utilization and seedling growth of wheat as affected by drought and salinity. Environmental and Experimental Botany, 55: 195-200.

22. Steppuhn, H., M.T. Van Genuchten and C. Grieve. 2005. Root-Zone Salinity. Crop Science, 45: 221 232.

23. Tahira, W.M., M. Ibrahim and A. Salam. 2006. Inheritance of salt tolerance as measured via root length in spring wheat (Triticum aestivum L.) under different salinity levels. Journal of Crop Improvement, 16: 131-139.

24. Tester, M. and R. Davenport. 2003. $\mathrm{Na}^{+}$tolerance and $\mathrm{Na}^{+}$transport in higher plants. Annals of Botany. 91: 503-527.

25. Tsegaye, D., D. Tadesse, D. Yigzaw and S. Getnet. 2012. Genetic variability, correlation and path analysis in durum wheat germplasm (Triticum durum). Agricultural Research and Reviews, 1: 107112.

26. Waines, J.G. and B. Ehdaie. 2007. Domestication and crop physiology: roots of green-revolution wheat. Annals of Botany, 100: 991-998.

27. Wittenmayer, L. and W. Merbach. 2005. Plant responses to drought and phosphorus deficiency: contribution of phytohormones in root-related processes. Journal of Plant Nutrition and Soil Science, 168: 531-540. 


\title{
Genetic Evaluation of Root Traits for Doubled Haploid Lines of Barely in Normal and Salt Stress Conditions
}

\section{Nasibeh Abdollahzadeh ${ }^{1}$, Reza Fotovat ${ }^{2}$, Farid Shekari ${ }^{3}$ and} Seyed Mohammad Alavi Siney

\author{
1- Graduate M.Sc., University of Zanjan, (Corresponding Author: abdollahzadehn@ yahoo.com) \\ 2- and 3- Assistant Professor and Associated Professor, University of Zanjan \\ 4- Faculty Member of Horticulture Crops Research Department, South Kerman Agricultural and Natural \\ Resources Research and Education Center, AREEO, Jiroft, Iran. \\ Received: November 24, $2014 \quad$ Accepted: March 1, 2015
}

\begin{abstract}
To evaluation of genetic parameters of rooting traits in 50 doubled haploid barley lines, a factorial experiment based on CRD was carried out at the agronomy lab of the Zanjan University. Analysis of variance showed significant differences for all rooting traits:material transfering speed, germination percent, angle, diameter, fresh and dry weight, surface area, cumulative length, the length of the longest root and the hairy root length. The calculated narrow sense heritability was medium for all studied rooting traits except dry root weight (15.11 and 23.40 in normal and stress conditions). Based on the genotypic correlations, it was found that there were positive and highly significant association normal and salt stress conditions, especialy between trait the length of the longest root and traits cumulative root length, root surface and fresh dry root weight. Since, the length of the longest root and cumulative root length and root surface offered as suitable traits for evaluating and selecting the genotypes in breeding programms.
\end{abstract}

Keywords: Barley, Genotypic Correlation, Narrow Sense Heritability, Salinity 